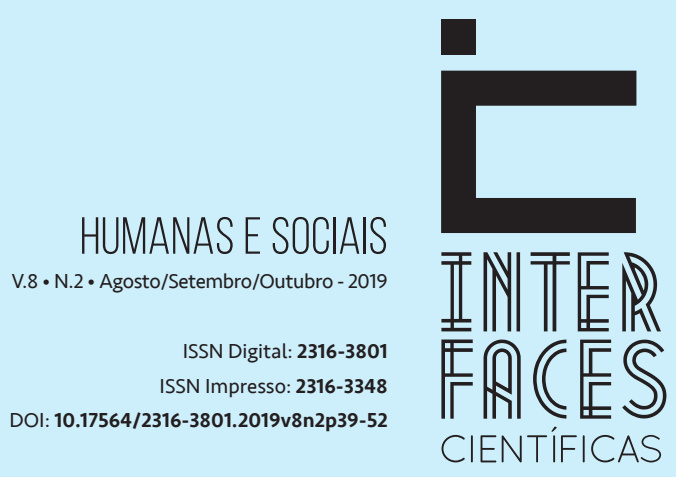

\section{A INTERFACE ENTRE DESIGN E SAÚDE: UMA REVISÃO BIBLIOGRÁFICA}

\section{THE DESIGN AND HEALTH INTERFACE: A BIBLLOGRAPHIC RESEARCH}

\section{LA INTERFAZ ENTRE DISEÑO Y SALUD: UNA REVISIÓN BIBLIOGRÁFICA}

\section{RESUMO}

Este artigo tem o objetivo de realizar uma revisão bibliográfica quantitativa em bases de dados científicos - Portal de Periódicos da CAPES, Scielo e Portal Regional da Biblioteca Virtual em Saúde (BVS) - no período de cinco anos (2011-2016). Foram coletados os artigos científicos nacionais relacionados à disciplina de design em interface com a área de saúde e suas referências bibliográficas analisadas. Os resultados apontam para uma disparidade quantitativa entre a produção científica brasileira e a internacional nesse eixo temático. 0 fato de o tema "saúde" não representar uma linha principal da pesquisa em design destaca o potencial de um campo emergente e promissor.

\section{PALAVRAS-CHAVE}

Design. Revisão Bibliográfica. Saúde 


\section{ABSTRACT}

In this article, we aim to realize a quantitative bibliographic research in scientific databases - Portal de Periódicos da CAPES (Portal of Periodicals CAPES), Scielo and Portal Regional da Biblioteca Virtual em Saúde (BVS, Regional Portal of the Virtual Health Library) - during the past five years (2011-2016). We collected the national scientific articles related to design in interface with health and analyzed their bibliographic references. The results indicate a quantitative disparity between the Brazilian and the international scientific production in this thematic axis. The fact that the subject "health" does not represent a major line of design research highlights the potential of an emerging and promising field.

\section{KEYWORDS}

Design. Bibliographic Research. Health

\section{RESUMEN}

Este artículo tiene como objetivo realizar una revisión bibliográfica cuantitativa en bases de datos científicos - Portal de periódicos de la CAPES, Scielo y Portal Regional de la Biblioteca virtual en Salud- en el lapso de cinco años (2011-2016). Fueron recogidos artículos científicos nacionales relacionados a la disciplina de diseño de interfaz en el área de salud y sus referencias bibliográficas analizadas. Los resultados apuntan a una desigualdad cuantitativa entre la producción científica brasileira e internacional en ese eje temático. El hecho de que el tema "salud" no representa una línea principal de investigación en diseño muestra el potencial de un campo emergente y promisorio.

\section{PALABRAS CLAVE}

Diseño. Revisión Bibliográfica. Salud 


\section{INTRODUÇ̧̃̃o}

A Organização Mundial de Saúde (OMS) propõe a seguinte definição para saúde: "um estado de completo bemestar físico, mental e social e não somente ausência de afecções e enfermidades" (SEGRE, 1997). Num mundo superpopuloso, complexo e repleto de adversidades socioeconômicas, alcançar essa condição torna-se um desafio cada vez mais difícil para a maioria das pessoas, fazendo desta preconização algo utópico e estatisticamente raro.

A saúde assegurada como "direito de todos e dever do Estado" é uma conquista do povo brasileiro promulgada na Constituição de 1988. Nesse mesmo ano foi também votada a criação do Sistema Único de Saúde (SUS), afirmando a universalidade, a integralidade e a equidade da atenção em saúde. No entanto, tanto no cenário brasileiro, quanto no panorama mundial, a garantia de saúde de qualidade para toda a população representa ainda um complicado desafio.

Para sanar as importantes lacunas dos serviços de saúde, políticas e diretrizes mais recentes conduzem à participação e integração de todos os envolvidos nesse contexto. O Ministério da Saúde (2004) elaborou um documento base para gestores e trabalhadores do SUS com propostas para instituir a Política Nacional de Humanização. Nesse relatório, todos os atores envolvidos nos serviços de saúde são convocados a participar e se responsabilizar pela gestão do sistema:

Por humanização entendemos a valorização dos diferentes sujeitos implicados no processo de produção de saúde: usuários, trabalhadores e gestores. Os valores que norteiam esta política são a autonomia e o protagonismo dos sujeitos, a corresponsabilidade entre eles, o estabelecimento de vínculos solidários e a participação coletiva no processo de gestão. (MINISTÉRIO DA SAÚDE, 2004, on-line).

Essa política tem por finalidade ampliar o debate sobre atenção à saúde, sensibilizar outros segmentos e, principalmente, tornar a humanização uma política pública de saúde (MINISTÉRIO..., 2004). Além dos trabalhadores e usuários diretamente implicados nos processos de promoção da saúde, inúmeros profissionais colaboram para a estruturação e manutenção do sistema. 0 design é uma profissão que desponta com grande potencial de atuação na área de saúde. Skrabe (2010) comenta que hoje o conceito de design é muito mais amplo, "incorpora a função, busca a boa estética por ser atributo humanizador, mas abarca todas as causas que produzem o resultado, assume compromisso com a sustentabilidade e mira os efeitos, que são seu propósito e sua razão de ser" (SKRABE, 2010, p. 78).

A interconexão entre a disciplina de design e a área de saúde representa, atualmente, um assunto de interesse internacional. Seja na esfera acadêmica ou no campo profissional, muitas são as possibilidades de ação dos designers. Sobre a complexidade dos desafios existentes na área de saúde e a inclusão promissora do design nesse contexto, Skrabe (2010, p. 108) pontua:

Esse novo contexto, de complexidade sem precedentes, cria um ponto de inflexão do setor. Só uma abordagem multidisciplinar, dotada de visão sistêmica, tem efetiva condição 
de reunir e articular todas as peças do gigantesco quebra-cabeças em que se converteu o hospital. E a resposta ao desafio abre espaço para o Design e sua vocação integradora. Abre espaço para um Design de resultados. Um Design baseado em evidência.

Este trabalho investiga a aplicação do design e suas competências em estudos científicos que envolvem de alguma forma a área de saúde. Contempla conceitos escolhidos em consonância com os interesses acadêmicos das autoras, sejam eles: Design Participativo, Design de Serviços e Design Centrado no ser humano.

\section{DESIGN DE SERVIÇOS}

Como disciplina, pode-se afirmar que o Design de Serviços teve início na década de 1990 e estruturou-se a partir de três núcleos de pesquisas: um na Alemanha, em Colônia, na Universidade de Ciências Aplicadas, criado pelos pesquisadores Michael Erlhoff e Birgit Mager; outro, no Reino Unido, com Gillian Hollins e Bill Hollins, na Escola de Negócios Westminster; e um terceiro, no Politécnico de Milão, na Itália, com Ézio Manzini (FREIRE, 2011).

Com as constantes mudanças no mundo, o setor de serviços tem ganhado uma importância cada vez maior para as organizações. Serviços são diferentes de produtos: acontecem ao longo do tempo, são complexos, interativos e desenvolvem-se em diferentes pontos de contato. Moritz afirma que o Design de Serviços não é uma nova especialidade da disciplina de design, mas sim uma plataforma multidisciplinar de conhecimentos. É o desenho de toda a experiência de um serviço, assim como do processo e da estratégia para fornecê-lo. O Design de Serviços é um processo contínuo de idealização e modelagem de serviços úteis, empregáveis, desejáveis, eficazes e eficientes (MORITZ, 2005).

Segundo Mager (2009), o Design de Serviços é um novo conceito que inaugura processos e métodos para os prestadores de serviços. Apresenta enfoque holístico que leva em consideração o contexto e os sistemas de relações e interações. Para contemplar esse tipo de abordagem, o trabalho do designer de serviços torna-se muitas vezes interdisciplinar. O Design de Serviços está vinculado à abordagem do Design Centrado no ser humano, devido à necessidade que possui de entender ou influenciar o comportamento de pessoas. Além disso, a cocriação também se faz presente quando o design deixa de enxergar os clientes como consumidores passivos e passa a integrá-los no processo de prestação de serviços como parceiros ativos (MAGER, 2009).

\section{DESIGN PARTICIPATIVO}

O desenvolvimento exponencial e o processo de globalização aos quais o mundo tem se submetido desde a década de 1950 modificaram as estruturas sociais e intensificaram as relações. Desde então, 
os conceitos de colaboração e participação, assim como suas aplicações, tem se tornado mais recorrentes em diversas áreas, sobretudo com as mudanças tecnológicas e de comunicação mais recentes.

No design, a colaboração tem ganhado destaque à medida que as atividades projetuais se tornam cada vez mais complexas e demandam abordagens interdisciplinares. O conceito de design, já sendo por natureza interdisciplinar, faz do design colaborativo apenas um impulsionador para uma interdisciplinaridade ainda mais profunda e sistematizada (MANZINI; VEZZOLI, 2008). Nele, agentes de diferentes áreas do conhecimento se engajam para produzir soluções que satisfaçam a todos.

Em 1971, o Design Participativo surge como uma especificação do conceito de colaboração focada na inclusão de um agente em especial, o usuário. Desde então, a prática do design evoluiu, o foco no usuário materializou-se e o padrão clássico do processo do design foi rompido. Neste último, o designer seria o especialista que cria ideias originais a partir das informações extraídas por um investigador, em pesquisas e estudos de mercado que focam o entendimento do usuário. Nas novas abordagens, todos os papéis estão conectados (STAPPERS; SLEESWIJK VISSER, 2007).

Conforme Moritz, o conceito principal do Design Participativo é inserir o usuário no contexto do projeto, tornando-o parte do processo de design. Essa nova forma de abordagem do design é particularmente relevante para o Design de Serviços (MORITZ, 2005).

\section{DESIGN CENTRADO NO SER HUMANO}

Para Giacomin, a inserção do usuário como um elemento-chave no processo de design representa uma mudança de ênfase na progressão do paradigma do design. Esse paradigma tem evoluído e prosperado ao longo dos anos, começando com a ergonomia e movendo-se para fatores humanos, usabilidade, design centrado no usuário, inclusão, design de interação, design empático, design de experiência do produto, design de experiência do cliente, design emocional, design emocional durável, branding sensorial, neurobranding, design de serviços e, por fim, mais recentemente, o Design Centrado no ser Humano (GIACOMIN, 2012).

Conforme explica Krippendorff (2000), o foco no ser humano surgiu nos anos 1950 na primeira mudança de paradigma, quando os produtos provenientes da era industrial, com características funcionais e fabricação em série, passaram a ser considerados bens de consumo, informação e identidade. Os designers começaram a compreender que os bens não eram só coisas, mas sim práticas sociais, preferências e símbolos, e que, ao projetar essas coisas intangíveis, deveriam não mais considerar a figura de usuários racionais, mas sim a de compradores, consumidores e determinados públicos.

Portanto, o Design Centrado no ser humano emerge da ruptura com uma visão que ignora as características humanas em benefício de fatores objetivos e passa a preocupar-se com a maneira com a qual as pessoas veem, interpretam e convivem com artefatos (KRIPPENDORFF, 2000).

A metodologia do Design Centrado no ser humano vem sendo praticada e pesquisada tanto no âmbito acadêmico quanto pelas empresas, em diversos setores. É um processo capaz de solucionar diversos tipos de problemas, desde o design de produtos e serviços até o design de espaços ou sis- 
temas. A aplicação dessa metodologia pode ser encontrada no setor de serviços, como descrito pela IDEO, uma empresa internacional de design e consultoria em inovação:

Para que um serviço seja eficaz, ele precisa ser considerado do início ao fim: desde como é divulgado até como é entregue. Para que um serviço tenha o impacto desejado, é essencial ter uma compreensão profunda das pessoas a quem você vai servir - não somente o que eles precisam ou desejam, mas quais limitações eles enfrentam, o que os motiva e o que é importante para eles. (IDEO, 2014, on-line)

O presente estudo realiza uma revisão bibliográfica quantitativa gerada por meio de um levantamento de produções científicas brasileiras publicadas em bases de dados e relacionadas à intervenção da disciplina de design na área de saúde.

As bases de dados consultadas foram selecionadas segundo alguns critérios. O Portal de Periódicos da CAPES foi a primeira base escolhida, por ser considerado o portal brasileiro de informação científica e uma base sólida e confiável. Por apresentar arquivos de qualidade, a segunda base de dados utilizada foi a da Scielo, uma biblioteca eletrônica que abrange uma coleção selecionada de periódicos científicos brasileiros. Por último, foi selecionado o Portal Regional da Biblioteca Virtual em Saúde (BVS), pelo fato de deter acesso diferenciado às melhores fontes de informação de evidência sobre os efeitos das intervenções em saúde nos países da América Latina e Caribe.

A pesquisa se restringiu a artigos completos, gratuitos e publicados nos últimos cinco anos (2011 a 2016), que possuem em seu título uma das combinações de palavras-chaves definidas para esse estudo conforme a Tabela 1.

\section{RESULTADOS}

Com base nos objetivos e interesses específicos das autoras dessa pesquisa, foram escolhidas quatro palavras-chave relacionadas à área de design: Design, Design de Serviços, Design Participativo e Design Centrado no ser humano. Essas palavras foram em seguida combinadas à palavra chave "Saúde". A Tabela 1 apresenta as palavras-chave e suas respectivas combinações com "Saúde”. Foram listadas as traduções correspondentes dessas palavras e de suas combinações na língua inglesa, principal idioma utilizado em pesquisa internacional (Tabela 2).

Tabela 1 - Palavras-chave escolhidas

\begin{tabular}{|c|c|}
\hline Palavras-chave & Combinações \\
\hline Design & Design e Saúde \\
\hline Design de Serviços & Design de Serviços e Saúde \\
\hline Design Participativo & Design Participativo e Saúde \\
\hline
\end{tabular}




\begin{tabular}{|c|c|}
\hline Design Centrado no ser humano & Design Centrado no ser humano e Saúde \\
\hline Saúde & \\
\hline
\end{tabular}

Fonte: Elaborado pelas autoras, com base na pesquisa realizada (2016).

Tabela 2 - Palavras-chave escolhidas traduzidas para o inglês

\begin{tabular}{|c|c|}
\hline Palavras-chave & Combinações \\
\hline Design & Design and Health \\
\hline Service Design & Service Design and Health \\
\hline Participatory Design & Participatory Design and Health \\
\hline Human-Centered Design & Human-Centered Design and Health \\
\hline Health & \\
\hline
\end{tabular}

Fonte: Elaborado pelas autoras, com base na pesquisa realizada (2016).

Estabelecidas as combinações de palavras-chave em português, foram pesquisados artigos completos cujos títulos detêm uma dessas junções e tenham sido publicados nos últimos cinco anos. Os resultados das pesquisas, utilizando as três bases de dados escolhidas estão especificados na Tabela 3.

Tabela 3 - Quantidade de artigos encontrados nas bases de dados que apresentam em seus títulos uma das combinações de palavras-chave definidas nesse estudo

\begin{tabular}{|c|c|c|c|c|}
\hline \multirow{2}{*}{ Base de dados } & \multicolumn{4}{|c|}{ Combinação de Palavras-Chave } \\
\cline { 2 - 5 } & Design e Saúde & $\begin{array}{c}\text { Design de Serviços } \\
\text { e Saúde }\end{array}$ & $\begin{array}{c}\text { Design } \\
\text { Participativo e } \\
\text { Saúde }\end{array}$ & $\begin{array}{c}\text { Design Centrado } \\
\text { no ser Humano e } \\
\text { Saúde }\end{array}$ \\
\hline CAPES & 24 & 0 & 0 & 1 \\
\hline SCIELO & 7 & 0 & 0 & 0 \\
\hline BVS & 19 & 1 & 0 & 0 \\
\hline Subtotal & 50 & 1 & 0 & 1 \\
\hline Total & \multicolumn{5}{|c|}{52} & \\
\hline
\end{tabular}

Fonte: Elaborado pelas autoras, com base na pesquisa realizada (2016).

Em um segundo momento, pesquisou-se artigos completos publicados de 2011 a 2016 que têm em seu título uma das combinações descritas nesse estudo e traduzida para o inglês. Os resultados obtidos nessa fase constituíram uma base para comparação quantitativa com a produção científica em português, notadamente brasileira (TABELA 4). 
Tabela 4 - Quantidade de artigos encontrados nas bases de dados que apresentam em seus títulos uma das combinações de palavras-chave definidas nesse estudo e traduzidas para o inglês

\begin{tabular}{|c|c|c|c|c|}
\hline \multirow{2}{*}{ Base de dados } & \multicolumn{4}{|c|}{ Combinação de Palavras-Chave } \\
\cline { 2 - 5 } & Design and Health & $\begin{array}{c}\text { Service Design and } \\
\text { Health }\end{array}$ & $\begin{array}{c}\text { Participatory } \\
\text { Design and Health }\end{array}$ & $\begin{array}{c}\text { Human-Centered } \\
\text { Design and } \\
\text { Health }\end{array}$ \\
\hline CAPES & 4.353 & 65 & 42 & 7 \\
\hline SCIELO & 8 & 0 & 0 & 0 \\
\hline BVS & 859 & 24 & 24 & 2 \\
\hline Subtotal & 5520 & 89 & 66 & 7 \\
\hline Total & & \multicolumn{4}{|c|}{5682} \\
\hline
\end{tabular}

Fonte: Elaborado pelas autoras, com base na pesquisa realizada (2016).

Nota-se que, utilizando-se a Tabela 4 como base comparativa, a produção científica internacional em língua inglesa é expressiva e bastante superior à produção nacional que se apresenta na Tabela 3.

Como resultado do processo de triagem, foram eliminados 44 artigos do total de 52 encontrados nas diferentes bases de dados. Excluiu-se 21, 5 e 17 artigos provenientes das bases CAPES, Scielo e BVS respectivamente, pelo fato de não possuírem uma das combinações de palavras-chave no título. Além disso, também ocorreu uma (1) repetição de artigo relevante na base BVS. Os trabalhos restantes, exibidos na Tabela 5, foram empregados para o embasamento teórico deste trabalho.

Tabela 5 - Artigos selecionados, respectivos autores principais e número de versões localizadas pela base de dados

\begin{tabular}{|c|c|c|c|}
\hline $\begin{array}{c}\text { Base de } \\
\text { dados }\end{array}$ & Título do artigo & Autor principal & $\begin{array}{c}\text { Número de } \\
\text { versões }\end{array}$ \\
\hline \multirow{6}{*}{ CAPES } & $\begin{array}{c}\text { Prevenção às DST/aids: design da informação para } \\
\text { promoção da saúde }\end{array}$ & FREITAS & 1 \\
\cline { 2 - 4 } & $\begin{array}{c}\text { Desenvolvimento e implementação de um am- } \\
\text { biente virtual de aprendizagem na área da saúde: } \\
\text { uma experiência de pesquisa baseada em design }\end{array}$ & $\begin{array}{c}\text { Fale com eles! O trabalho interpretativo e a produ- } \\
\text { ção de consenso na pesquisa qualitativa em saú- } \\
\text { de: inovações a partir de desenhos participativos }\end{array}$ & ONOCKO-CAMPOS \\
\cline { 2 - 4 } & $\begin{array}{c}\text { A criação de estratégia de comunicação para } \\
\text { prevenção em saúde através do design centrado } \\
\text { no ser humano }\end{array}$ & 3 \\
\hline \multicolumn{2}{|c}{ FREIRE } & 1 \\
\hline
\end{tabular}




\begin{tabular}{|c|c|c|c|}
\hline $\begin{array}{c}\text { Base de } \\
\text { dados }\end{array}$ & Título do artigo & Autor principal & $\begin{array}{c}\text { Número de } \\
\text { versões }\end{array}$ \\
\hline \multirow{2}{*}{ Scielo } & $\begin{array}{c}\text { Desenvolvimento e implementação de um am- } \\
\text { biente virtual de aprendizagem na área da saúde: } \\
\text { uma experiência de pesquisa baseada em design }\end{array}$ & STRUCHINER & 1 \\
\cline { 2 - 4 } & $\begin{array}{c}\text { Fale com eles! O trabalho interpretativo e a produ- } \\
\text { ção de consenso na pesquisa qualitativa em saú- } \\
\text { de: inovações a partir de desenhos participativos }\end{array}$ & ONOCKO-CAMPOS & 1 \\
\hline \multirow{2}{*}{ BVS } & $\begin{array}{c}\text { Desenvolvimento e implementação de um am- } \\
\text { biente virtual de aprendizagem na área da saúde: } \\
\text { uma experiência de pesquisa baseada em design }\end{array}$ & STRUCHINER & 2 \\
\cline { 2 - 4 } & $\begin{array}{c}\text { Fale com eles! O trabalho interpretativo e a produ- } \\
\text { ção de consenso na pesquisa qualitativa em saú- } \\
\text { de: inovações a partir de desenhos participativos }\end{array}$ & ONOCKO-CAMPOS & 1 \\
\hline
\end{tabular}

Fonte: Elaborado pelas autoras, com base na pesquisa realizada (2016).

Ao serem suprimidos os artigos duplicados, obtém-se o reduzido número de quatro (4) trabalhos científicos brasileiros que empregaram conhecimentos e competências da área de design ao campo de saúde.

Nesses quatro (4) artigos remanescentes foram identificados 81 referências bibliográficas destas sete (7) são referências de sites (sem autor definido), 50 possuem autorias diferentes entre si e em 24 bibliografias 11 autores se repetem.

Para aprofundar o estudo, as referências bibliográficas foram extraídas dos artigos selecionados e os respectivos autores listados. Uma relação de autores ordenada alfabeticamente foi elaborada e eliminou-se as referências duplicadas. Por último, a lista foi reordenada por número de aparições.

Com base nessa relação de referências bibliográficas, a Tabela 6 apresenta os autores mais citados e o número de vezes que aparecem, como também, os mais relevantes.

Tabela 6 - Artigos selecionados, respectivos autores mais citados e número de aparições de cada autor

\begin{tabular}{|c|c|c|}
\hline Título do artigo & Autores mais citados & $\begin{array}{c}\text { Número de } \\
\text { citações }\end{array}$ \\
\hline $\begin{array}{c}\text { Prevenção às DST/aids: design da informação } \\
\text { para promoção da saúde }\end{array}$ & BONSIEPE & 2 \\
\cline { 2 - 3 } & BARDIN & 2 \\
\hline $\begin{array}{c}\text { Desenvolvimento e implementação de um ambiente virtual } \\
\text { de aprendizagem na área da saúde: uma experiência de } \\
\text { pesquisa baseada em design }\end{array}$ & BARAB e SQUIRE & 1 \\
\hline
\end{tabular}




\begin{tabular}{|c|c|c|}
\hline Título do artigo & Autores mais citados & $\begin{array}{c}\text { Número de } \\
\text { citações }\end{array}$ \\
\hline $\begin{array}{c}\text { Fale com eles! O trabalho interpretativo e a produção de con- } \\
\text { senso na pesquisa qualitativa em saúde: inovações a partir } \\
\text { de desenhos participativos }\end{array}$ & GADAMER & 3 \\
\cline { 2 - 3 } $\begin{array}{c}\text { A criação de estratégia de comunicação para prevenção em } \\
\text { saúde através do design centrado no ser humano }\end{array}$ & FREUD & 3 \\
\hline
\end{tabular}

Fonte: Elaborado pelas autoras, com base na pesquisa realizada (2016).

Dentre estes autores destaca-se Gui Bonsiepe, um dos mais renomados designers e teóricos do mundo, pioneiro em diferentes campos da teoria do design e a autora Laurence Bardin, que se situa, atualmente, como literatura de referência em "análise de conteúdo".

\section{DISCUSSÃO}

A maioria dos autores citados na fundamentação teórica deste artigo não é mencionada em nenhum dos trabalhos científicos relacionados. Bardin (2009) trata da Análise de Conteúdo, uma metodologia que a autora empregou na investigação psicossociológica e nos estudos das comunicações de massas. Os autores Barab e Squire (2004) discutem a pesquisa baseada em Design (Design-Based Researh - DBR, em inglês) e não consideram essa uma metodologia, mas sim várias abordagens de pesquisa desenvolvidas em contextos reais, nas quais é possível utilizar métodos qualitativos e quantitativos. Gadamer (1997) aborda a hermenêutica filosófica, ou seja, a metodologia das ciências do espírito e da natureza na busca da verdade, à luz da ciência da hermenêutica. Em “Construções em análise” (1937), Freud discorre sobre a técnica psicanalítica da construção.

Bonsiepe (2011) é o único autor citado no referencial teórico deste artigo e em dois dos trabalhos encontrados na revisão bibliográfica produzida. Sua obra "Design, Cultura e Sociedade" (2011) apresenta uma análise sobre a evolução do design ao longo do século XX, assinalando seus sucessos e desvios. Dentre os autores mais citados, somente Barab, Squire e Bonsiepe atuam na área de design, resultado que nos faz refletir sobre a incipiência da contribuição da prática projetual para a área de saúde.

A última consideração a ser feita diz respeito à discrepância do volume de produções científicas brasileiras em comparação com a contribuição internacional. Esse resultado chama atenção para a potencialidade de um campo emergente e pioneiro na interface entre design e saúde. Propensa a possibilidades inovadoras, a junção desses conhecimentos pode resultar no desenvolvimento de projetos e ações promissoras para todos os envolvidos.

\section{CONCLUSÃO}


Este artigo descreve a realização de uma revisão bibliográfica quantitativa para investigar a aplicação do design em estudos científicos vinculados à área de saúde. Para iniciar a pesquisa, os seguintes conceitos pertinentes às intenções acadêmicas das autoras foram escolhidos: Design de Serviços, Design Participativo e Design Centrado no ser humano. A partir desses conceitos e do termo "Saúde", quatro combinações foram preparadas como expressões de busca. Em seguida, utilizou-se três bases de dados para fazer um levantamento das produções brasileiras: Portal de Periódicos da CAPES, Scielo e Portal Regional da BVS.

A busca inicial apontou 52 artigos que, após seguidas triagens, reduziram-se a quatro (4) artigos pertinentes a esta revisão bibliográfica. Todas as bases de dados apresentaram pelo menos dois desses artigos, sendo que o Portal de Periódicos da Capes continha os quatro.

Nesses quatro artigos foram identificadas 81 referências bibliográficas, com 61 autores diferentes, e destes 11 se repetem. Os autores mais relevantes para este trabalho são Bonsiepe, Barab e Squire, dado o fato de exercerem atividades na área de design.

A revisão bibliográfica permitiu localizar artigos que abordam assuntos relacionados à interação da disciplina de design com o campo da saúde. Nota-se que essa temática não parece ser amplamente abordada no cenário brasileiro, sobretudo em comparação com a expressividade das produções internacionais. A interação entre essas duas áreas do conhecimento mostra-se como um campo promissor a ser explorado por pesquisadores e estudiosos na procura por soluções inovadoras.

\section{REFERÊNCIAS}

BARAB, Sasha; SQUIRE Kurt. Design-based research: putting a stake in the ground. J learn Sci., v. 13, n. 1, p. 1-14, 2004.

BARDIN, Laurence. Análise de Conteúdo. Tradução Luís Antero Reto e Augusto Pinheiro. Lisboa: Edições 70, 2009.

BONSIEPE, Gui. Design, cultura e sociedade. São Paulo: Blucher, 2011.

FREIRE, Karine de Mello. Design de serviços, comunicação e inovação social: um estudo sobre serviços de atenção primária à saúde. 2011. 254f. Tese (Doutorado) - Programa de Pós-Graduação de Artes \& Design, Pontifícia Universidade Católica do Rio de Janeiro (PUC-RIO), Rio de Janeiro, 2011.

FREUD, Sigmund. Construções em análise. Edição standard brasileira das obras psicológicas completas de Sigmund Freud. Rio de Janeiro: Imago, 1975 [1937].

GADAMER, H-G. Verdade e método: traços fundamentais de uma hermenêutica filosófica. Petrópolis: Vozes, 1997. 
GIACOMIN, Joseph. What is Human Centred Design? Congresso Brasileiro de Pesquisa em Design, 10, São Luís - MA, Anais..., São Luís: EDUFMA, 2012, p.148-161.

IDEO - An Introduction to Human-Centered Design: The Design Process. 2014. Disponível em: http:// plusacumen.org/wpcontent/uploads/2014/04/Class_1_readings.pdf. Acesso em: 17 dez. 2016.

KRIPPENDORFF, Klaus. Propositions of Human-centeredness: A Philosophy for Design. In: DURLING, D.; FRIEDMAN, K. (Eds.). Doctoral Education in Design: Foundations for the Future. Staffordshire (UK): Staffordshire University Press, 2000. p.55-63.

MAGER, Birgit. Service Design as an emerging field. In: MIETTINEN, Satu; KOIVISTO, Mikko (Ed.). Designing Services with innovative methods. Keuruu: University of Art and Design Helsinki, v. 1, p. 28-43, 2009.

MANZINI, Ezio; VEZZOLI, Carlo. 0 desenvolvimento de produtos sustentáveis: Os requisitos ambientais dos produtos industriais. São Paulo: Edusp, 2008.

MINISTÉRIO DA SAÚDE - Política nacional de humanização. Brasília: Ministério da Saúde, 2004. Disponível em: http://www.crpsp.org.br/diverpsi/arquivos/pnh-2004.pdf. Acesso em: 18 dez. 2016.

MORITZ, Stefan. Service Design: pratical acess to an evolving field. Köln International School of Design, University of Applied Sciences Cologne, 2005. 125 p.

SEGRE, Marco. O Conceito de saúde. Revista Saúde Pública, São Paulo, v. 31, n. 5, p. 538-542. 1997.

SKRABE, Celso. Chegou a hora e a vez do design. Anuário Hospital Best. Eximia Comunicação: São Paulo, 2010. Disponível em: https://issuu.com/fdellatorre/docs/anuario_design_01_montado. Acesso em: 18 dez. 2016.

STAPPERS, P. J.; SLEESWIJK VISSER, F. Bringing participatory design techniques to industrial design engineers. Engineering and Product Design Education Conference, NewCastle, p. 117-122, 2007. Disponível em: http://studiolab.io.tudelft.nl/manila/gems/sleeswijkvisser/epdecm.pdf. Acesso em: 17 dez. 2016. 


\section{Como citar este artigo:}

ROMEO, Andrea. Lo special account del fenomeno religioso nel dibattito nordamericano. Argumenta Journal Law, Jacarezinho - PR, Brasil, n. 29., 2018, p. 15-48. DOI: $10.17564 / 2316-3828.2018 v 7 n 1 p 13-24$

1 Graduada em Engenharia Mecânica pela Universidade Federal de Itajubá (2011) - UNIFEI e pela École Nationale d'Ingénieurs de Metz - ENIM (2011); Mestranda do Programa de Pós-graduação em Design, Inovação e Sustentabilidade da Universidade do Estado de Minas Gerais - UEMG. E-mail: rdmanza@gmail.com

2 Doutora (1997) e pós-doutor em Química pela Universidade Federal de Minas Gerais - UFMG; Graduada em Geologia (1977) pela (UFMG); PhD em Engineering Materials pela University of Sheffield (1994); Coordenadora Executiva do Programa de Pós-graduação em Engenharia de Materiais - REDEMAT; Professora permanente da Universidade do Estado de Minas Gerais (UEMG); Integrante do Núcleo de Estudos, Teoria, Pesquisa e Cultura em Design da UEMG.. E-mail: sebastiana.lana@gmail.com 
